\title{
Immunomodulatory Oligonucleotide HYB2055
}

National Cancer Institute

\section{Source}

National Cancer Institute. Immunomodulatory Oligonucleotide HYB2055. NCI

Thesaurus. Code C49182.

A second generation synthetic oligonucleotide with immunomodulatory and potential antineoplastic activities. HYB2055 consists of unmethylated CpG dinucleotide motifs that are present abundantly in bacterial and parasitic DNA, and a novel DNA structure, called an immunomer that contributes to metabolic stability of the agent. Upon infections, CpGcontaining DNA released from pathogenic organisms triggers host immune responses, which are mediated by the action of intracellular toll-like receptor 9 (T LR9), a pattern recognition receptor. This agent mimics bacterial DNA and selectively activates TLR9, thereby initiating immune signaling pathways, and leading to activation of B-cells and dendritic cells and induction of Th1-type cytokine production. 